\title{
Correlation of Blood FoxP3+ Regulatory T Cells and Disease Activity of Atopic Dermatitis
}

\author{
Yan Li $\mathbb{D}^{1},{ }^{1}$ Wei Xu $\left(\mathbb{D},{ }^{1}\right.$ Jingyi Yao, ${ }^{2,3,4}$ Haiyan Cheng $\mathbb{D}^{1},{ }^{1}$ Xiaoli Sun, ${ }^{1}$ and Linfeng Li $\mathbb{D}^{1}$ \\ ${ }^{1}$ Department of Dermatology, Beijing Friendship Hospital, Capital Medical University, Beijing 100050, China \\ ${ }^{2}$ Research Center of Beijing Friendship Hospital, Capital Medical University, China \\ ${ }^{3}$ Beijing Institute of Clinical Medicine, China \\ ${ }^{4}$ Beijing Key Laboratory of Transplant Tolerance and Organ Protection, Beijing 100050, China
}

Correspondence should be addressed to Linfeng Li; zoonli@sina.com

Received 14 February 2019; Accepted 30 August 2019; Published 15 September 2019

Academic Editor: Paulina Wlasiuk

Copyright @ 2019 Yan Li et al. This is an open access article distributed under the Creative Commons Attribution License, which permits unrestricted use, distribution, and reproduction in any medium, provided the original work is properly cited.

Objectives. To investigate CD4+CD25+FoxP3+ T regulatory cells (Tregs) in the peripheral blood of patients with atopic dermatitis (AD) and its correlation with disease severity. Methods. Blood samples from 79 AD patients before and after four-week conventional treatment were collected. Cell counts of CD4+CD25+FoxP3+Tregs, CD4+CD25+FoxP3-T effector cells (Teffs), and CD4+IL-10+Tregs were analyzed by flow cytometry. Serum levels of IL-4, IL-10, IL-12, IL-13, IFN- $\gamma$, and TGF- $\beta$ were measured by ELISA. Results. The pretreatment cell count of CD4+CD25+FoxP3+Tregs positively correlated with disease severity in all patients $(P<0.0001)$. However, when that correlation was rechecked based on the treatment response, a much stronger correlation of that was found in those patients with remission after treatment, while no correlation of that was found in patients without remission. Both the cell count and proportions of peripheral CD4+CD25+FoxP3+Tregs and CD4+CD25+FoxP3-Teffs reduced significantly after treatment in patients with remission, but remained unchanged in patients without remission. The cell count and proportion of CD4+IL-10+Tregs did not change after treatment in both groups. In patients with remission, serum levels of IL-4 and IL-13 significantly reduced (all $P<0.05$ ); IL-12 and IFN- $\gamma$ levels increased significantly (all $P<0.05$ ); IL-10 and TGF- $\beta$ levels remained unchanged after treatment. None of those cytokine levels changed in patients without remission. Conclusions. CD4+CD25+FoxP3+Tregs is associated with AD development and severity in some patients but not in others. AD maybe divided into CD4+CD25+FoxP3+Treg-associated subtype, which CD4+CD25+FoxP3+Treg is parallel to the activity of $\mathrm{AD}$, and nonassociated subtype, which $\mathrm{CD} 4+\mathrm{CD} 25+\mathrm{FoxP} 3+$ Treg is not related. This subgroup difference may contribute partly to the nonidentical markers that have been found in $\mathrm{AD}$ and should be studied further.

\section{Introduction}

Atopic dermatitis (AD), also called atopic eczema, is a chronic, recurrent, and inflammatory skin disease. It is characterized by severe pruritus, eczema-like lesions, and dry skin [1]. The etiology of AD is complex. In spite of intensive and extensive efforts in research, the exact etiology remains unclear. Recent studies suggest that it may be associated with immune disorder, such as a Th1/Th2-associated chemokine imbalance and skin barrier dysfunction $[2,3]$. In addition, patient genetics and environment factors could also contribute to the initiation and development of $\mathrm{AD}$. Most research on the etiology of AD emphasizes the correla- tion between immune disorders and AD. Previous studies have shown that immunoglobulin E- (IgE-) mediated allergic reaction results in inflammatory skin responses that are similar to the skin lesions of AD [4]. Disruption of the homeostasis of the effector T cells (Teffs) and regulatory T cells (Tregs) is considered a critical etiological factor for AD. It is well accepted that Tregs regulate autoimmune response and play essential roles in many autoimmune, allergic, and inflammatory diseases [5]. Tregs can prevent excessive immune response by inhibiting the activity and proliferation of Teffs so to enhance immune tolerance and maintain immune homeostasis. Thus, Tregs are critical for maintaining peripheral tolerance, preventing autoimmune diseases, and limiting 
inflammatory responses $[5,6]$. Tregs include natural Tregs and induced Tregs. CD4+CD25+FoxP3+Tregs belong to natural Tregs and overexpress forkhead box P3 (Foxp3), which is essential for $\mathrm{CD} 4+\mathrm{CD} 25+$ Treg function. Activated CD4+CD25+Tregs can release anti-inflammatory cytokines, such as TGF- $\beta$ and IL-10. Although the cytokines may be involved in the function of CD4+CD25+Tregs, they are not required for $\mathrm{CD} 4+\mathrm{CD} 25+$ Tregs-mediated regulation of immune function; instead, a direct cell-cell contact is required $[6,7]$. $\mathrm{CD} 4+\mathrm{CD} 25+\mathrm{FoxP} 3+$ Tregs inhibit the function of antigen-presenting cells (APC), effector T cells, and mast cells [5]. Induced Treg such as $\operatorname{Tr} 1$ cells overexpress IL-10. Although these T cells do not express Foxp3, they can upregulate Foxp3 after being activated. CD4+IL$10+$ Tregs release IL-10 to inhibit the proliferation of Th1 and Th2 cells [8]. Natural Tregs and induced Tregs have very different gene expression profiles and different functions [9]. The specific types, quantity, and precise function of Tregs in patients with $\mathrm{AD}$ are still unclear [10], and previous findings on Tregs of patients with $\mathrm{AD}$ are controversial [11-13]. To investigate the association between $\mathrm{AD}$ and CD4+CD25+FoxP3+Tregs, we collected peripheral blood samples from patients with moderate to severe AD before and after treatment and analyzed Treg characteristics.

\section{Materials and Methods}

2.1. Study Design. This was a prospective observational cohort study. The study protocol has been approved by the Institutional Review Board of Beijing Friendship Hospital (Approval No. 2015-P2-066-01). Written informed consent was obtained from each study participant.

2.2. Patients. Consecutive patients with moderate to severe $\mathrm{AD}$ treated in the Department of Dermatology of Beijing Friendship Hospital from January 1 to December 31, 2016 were included. The diagnosis of AD was confirmed using the UK Working Party's diagnostic criteria. The severity of $\mathrm{AD}$ was assessed based on the Rajka and Langeland criteria. At least 10\% body surface was affected by the disease. Patients with the eczema area and severity index (EASI) [14] between 7 and 14 were considered moderate to severe AD. Patients, who were pregnant, had autoimmune diseases, chronic systemic disease, and/or other skin diseases, or had a history of tumor or familial tumor diseases were excluded.

All patients were treated with conventional therapies for $\mathrm{AD}$, including antihistamines such as cetirizine tablets, loratadine, and mizolastine, compound glycyrrhizin capsules, topical mometasone furoate cream, and emollients for four weeks. Treatment effectiveness index $=($ pretreatment EASI posttreatment EASI)/pretreatment EASI $\times 100 \%$. Complete cure was defined as treatment effectiveness index $\geq 90 \%$. Significant improvement was defined as $60 \% \leq$ treatment effectiveness index $<90 \%$. Moderate improvement was defined as $20 \% \leq$ treatment effectiveness index $<60 \%$. No improvement was defined as treatment effectiveness index $<20 \%$ [15]. Patients with complete cure or significant improvement were considered to have AD remission. Patients with mod- erate improvement or no improvement were considered to have active $\mathrm{AD}$.

2.3. Flow Cytometry. Peripheral venous blood was collected from patients before and after treatment. Peripheral blood mononuclear cells (PBMCs) were extracted from the blood samples and stored at $-80^{\circ} \mathrm{C}$. After all samples collected, PBMCs were washed twice with DPBS (Corning, USA) and then resuspended in RPMI 1640 (Corning, USA) with 2\% FBS (Gibco, USA). Then, the suspended cells were stimulated by cell stimulation cocktail (eBioscience, USA) for 4 hours in $37^{\circ} \mathrm{C}$ with $5 \% \mathrm{CO}_{2}$. After stimulation, cells were washed and incubated with anti-CD4-FITC, anti-CD4-Percp, anti-CD25-PE, and anti-CD127-FITC. All the aforementioned reagents were purchased from $\mathrm{BD}$ Biosciences (USA). Intracellular IL-10-PE staining was performed using Transcription Factor Buffer Set (BD Biosciences, USA), and FOXP3-647 staining was performed using the Human FoxP3 Buffer Set (BD Biosciences, USA) according to the manufacturer's instructions. Data were collected using a four-color FACSCalibur ${ }^{\mathrm{TM}}$ flow cytometer (BD Biosciences, USA) and analyzed using FlowJo software version 10.1 (Tree Star, Ashland, OR, USA).

2.4. ELISA. The levels of IFN- $\gamma$, IL-4, IL-10, IL-12, IL-13, and TGF- $\beta$ in the serum samples were analyzed by ELISA using ELISA kits. The ELISA kits for human IL-4 (Catalog \#KHC0041), human IL-10 (Catalog \#KHC0101), human IL-12 p40/p70 (Catalog \#KHC0121), human IL-13 (Catalog \#KHC0131), and Human IFN- $\gamma$ (Catalog \#KHC4021) were from Invitrogen (USA). The ELISA kit for human TGFB2 was from Thermo Scientific (USA). ELISA reader (Spectra Max M3) was from Molecular Devices (USA).

2.5. Statistical Analysis. Descriptive statistics were used to detail the baseline characteristics. Mean with standard deviation (SD) and range was used for continuous variables (age, disease history, and EASI before and after treatment). Absolute number was used for categorical variables (gender). A paired $t$-test was used to compare different types of $\mathrm{T}$ cells $(\mathrm{CD} 4+\mathrm{CD} 25+\mathrm{FoxP} 3+$ Tregs, CD4+CD25+FoxP3Teffs, CD4+IL-10+Tregs) and cytokine levels before and after treatment of the $\mathrm{AD}$ remission and active groups. The association between CD4+CD25+FoxP3+Tregs count and pretreatment EASI for the two groups was analyzed by Pearson's correlation test.

All statistical analyses were conducted using SPSS version 16.0. $P<0.05$ was considered statistically significant.

\section{Results}

3.1. General Clinical Data. A total of 79 patients with moderate to severe $\mathrm{AD}$ were included. Of the 79 patients (aged 1940 years, 33 men), 40 became $\mathrm{AD}$ remission after treatment; 39 remained active AD after treatment. Patients' general clinical data are presented in Table 1. The mean age of the 79 patients was $25.06 \pm 5.20$ years. Demographics and pretreatment EASI were similar in the remission and active groups (Table 1). 
TABLE 1: General clinical data.

\begin{tabular}{lcc}
\hline & $\begin{array}{c}\text { AD remission } \\
\text { after treatment } \\
(N=40)\end{array}$ & $\begin{array}{c}\text { AD active } \\
\text { after treatment } \\
(N=39)\end{array}$ \\
\hline $\begin{array}{l}\text { Men/women } \\
\text { Age }\end{array}$ & $13 / 27$ & $20 / 19$ \\
$\quad$ Range, min-max (years) & $20-38$ & $19-40$ \\
$\quad$ Mean \pm SD (years) & $25.25 \pm 5.19$ & $24.87 \pm 5.27$ \\
Disease history (months) & & \\
$\quad$ Range, min-max (months) & $2-85$ & $3-76$ \\
$\quad$ Mean \pm SD (months) & $19.18 \pm 20.56$ & $18.92 \pm 15.33$ \\
EASI & & \\
$\quad$ Before treatment & & \\
$\quad$ Range, min-max & $7.67-13.31$ & $8.73-13.23$ \\
$\quad$ Mean $\pm S D$ & $10.29 \pm 1.19$ & $10.43 \pm 1.04$ \\
After treatment & & \\
$\quad$ Range & $2.23-4.98$ & $5.98-8.45$ \\
$\quad$ Mean $\pm S D$ & $3.61 \pm 0.64$ & $6.80 \pm 0.60$ \\
\hline
\end{tabular}

3.2. Comparison of Different Types of T Cell Counts before versus after Treatment. The pretreatment cell count of CD4+CD25+FoxP3+Tregs positively correlated with their pretreatment EASI in all AD patients $(r=-0.482, P \leq$ 0.001 , Figure 1(a)). Subsequently, these AD patients were divided into remission group and still active group according to their response to the treatment effect. Notably, the pretreatment $\mathrm{CD} 4+\mathrm{CD} 25+\mathrm{FoxP} 3+$ Treg cell count of patients from the $\mathrm{AD}$ remission group linearly correlated to their pretreatment EASI significantly $(r=0.95, \quad P<0.0001$, Figure 1(b)), whereas no such correlation was observed in patients from the $\mathrm{AD}$ active group (Figure 1(c)). Mean cell count and proportions of different types of $\mathrm{T}$ cells are presented in Table 2. For patients in the $\mathrm{AD}$ remission group, both the absolute cell counts and the proportions of CD4+CD25+FoxP3+Tregs and CD4+CD25+FoxP3-Teffs reduced significantly after treatment (all $P<0.05$, Table 2 ). In contrast to the $\mathrm{AD}$ remission group, patients in the $\mathrm{AD}$ active group showed similar cell counts and proportions of those $\mathrm{T}$ cells before and after treatment (Table 2). Moreover, the cell count and proportion of CD4+IL-10 Tregs were not significantly changed by treatment in both $\mathrm{AD}$ remission and active groups (Table 2). Representative scatter plots of analyses for CD4+CD25+FoxP3+Tregs (Figure 2), CD4+ CD25+FoxP3-Teffs (Figure 3), and CD4+IL-10+Tregs (Figure 4) are displayed in Figures 2-4.

3.3. Comparison of Cytokine Levels in Serum. For patients in the $\mathrm{AD}$ remission group, compared with the values before treatment, posttreatment serum levels of IL- 4 and IL-13 reduced significantly (all $P<0.05$ ), IFN and IL-12 levels increased significantly (all $P<0.05$ ), and IL-10 and TGF- $\beta$ levels remained unchanged (Figure 5). In contrast to the $\mathrm{AD}$ remission group, none of those cytokines were significantly changed after treatment in patients from the $\mathrm{AD}$ active group (Figure 5).

\section{Discussion}

The current study found that the pretreatment number of CD4+CD25+FoxP3+Tregs was associated with $\mathrm{AD}$ severity and reduced significantly after treatment in the remission group, whereas the active group did not show this association. These findings suggest that $\mathrm{AD}$ may have a CD4+CD25+FoxP3+Treg-associated subtype and a nonassociated subtype.

Previous studies on $\mathrm{CD} 4+\mathrm{CD} 25+$ Tregs in $\mathrm{AD}$ have shown controversial results. In 2004, Ou et al. reported that the cell count of $\mathrm{CD} 4+\mathrm{CD} 25+$ Tregs in the peripheral blood of patients with $\mathrm{AD}$ was twice of that of the normal control group [11]. However, in 2009, the two independent research groups Szegedi et al. and Brandt et al. found that the cell count of CD4+CD25+FoxP3+Tregs of patients with $\mathrm{AD}$ was not different from that of the healthy controls $[16,17]$. Notably, more recent studies demonstrated that patients with $\mathrm{AD}$ showed increased $\mathrm{CD} 4+\mathrm{CD} 25+\mathrm{FoxP} 3+$ Treg cell count and the cell count positively correlated with $\mathrm{AD}$ severity $[10,11,18,19]$. Our results showing the posttreatment reduction in the numbers of CD4+CD25+FoxP $3+$ Tregs are consistent with those of the more recent studies. We believe the conflicting findings may indeed reflect the complex roles of CD4+CD25+FoxP3+Tregs in the initiation and development of $\mathrm{AD}$. Our results also indicate that $\mathrm{CD} 4+\mathrm{CD} 25+\mathrm{FoxP} 3+$ Tregs could be involved in $\mathrm{AD}$ development only in some patients. Elevation of circulating Tregs could be a compensatory mechanism for the severe inflammation of patients with $\mathrm{AD}[20]$.

Tregs induce, maintain, and inhibit Th1/Th2 immune responses via Th1-type and Th2-type cytokines [21]. Thus, we determined these cytokine levels in serum. IL-4 and IL13 can stimulate B cell proliferation and IgE synthesis so to induce $\mathrm{T}$ cells to differentiate into Th2 cells and inhibit Th1 differentiation. We found that serum IL-4 and IL-13 levels were reduced after treatment in the remission group. Thus, IL-4 and IL-13 may be the essential Th2-type cytokines to induce $\mathrm{AD}$ and play critical roles in the etiology of acute and chronic $\mathrm{AD}$ in some patients $[1,22,23]$. IFN- $\gamma$ and IL12 , which are mainly secreted by Th1 cells and APCs, can induce $\mathrm{NK}$ and $\mathrm{T}$ cell proliferation, promote $\mathrm{T}$ and $\mathrm{NK}$ cell-mediated cytotoxicity, stimulate Th0-to-Th1 differentiation, regulate Th1 cell proliferation, and inhibit IgE synthesis and Th2 function. Our current study showed serum IFN- $\gamma$ and IL-12 levels were increased after treatment in the remission group. Previous studies on serum IL-10 levels in patients with AD show inconsistent results. Vakirlis et al. found that serum IL-10 levels in patients with $\mathrm{AD}$ were lower than those in the healthy controls [24]. However, Lesiak et al. reported that PBMCs of patients with AD produced more IL-10 than the PBMCs of healthy controls [25]. Hussein et al. found no significant difference in serum IL-10 levels between patients with $\mathrm{AD}$ and the healthy controls [26]. We found no changes in serum IL-10 after treatment in our patients. The inconsistent results on serum IL-10 levels in AD may indirectly reflect the complex mechanism underlying IL-10 production in the human body. In addition to Th2 cells, Th0, Trl, and B cells also can release IL-10 [27]. 


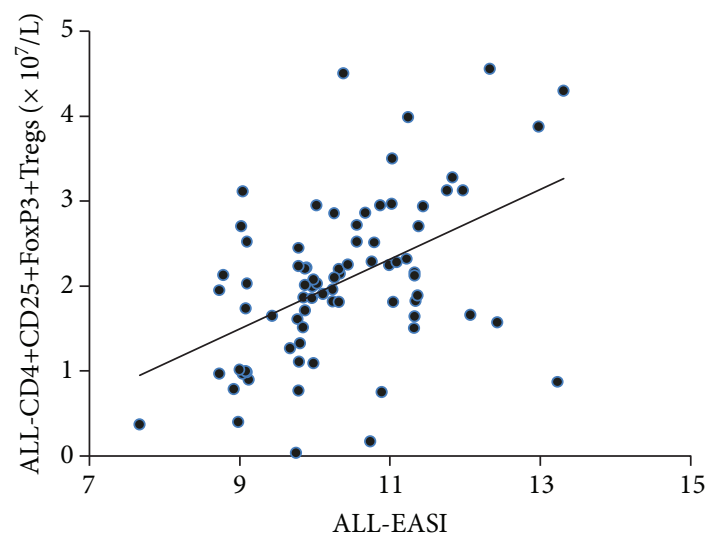

(a)

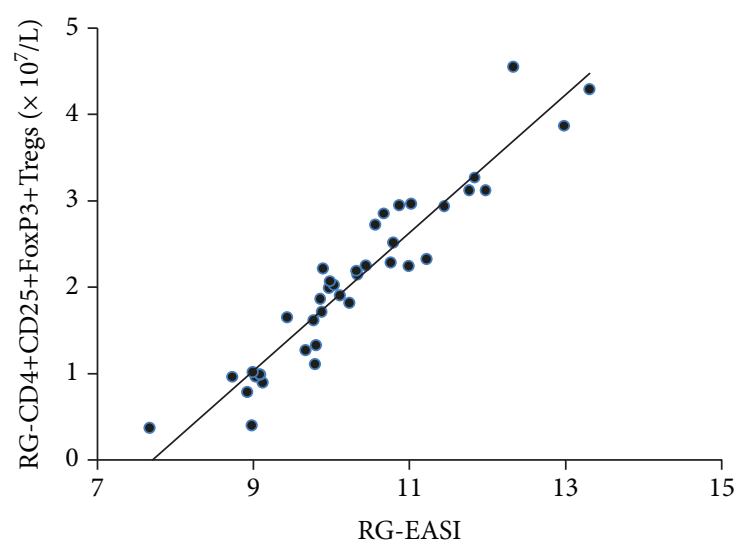

(b)

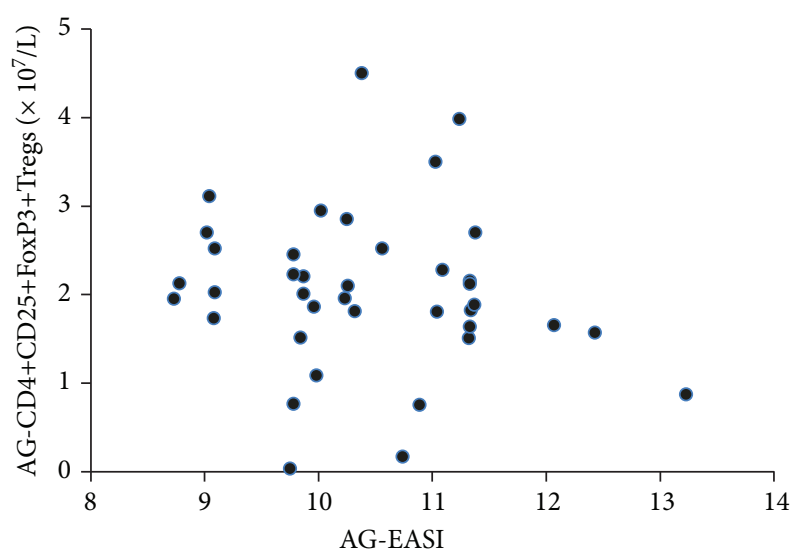

(c)

FIGURE 1: Correlation between pretreatment CD4+CD25+FoxP3+Treg count and EASI. (a) The pretreatment cell count of CD4+CD25+ FoxP3+Tregs positively correlated with their pretreatment EASI in all AD patients. (b) Patients in the AD remission group (RG) showed significant linear correlation between their pretreatment CD4+CD25+FoxP3+Treg count and pretreatment EASI. (c) No such linear correlation in the $\mathrm{AD}$ active group $(\mathrm{AG})$.

TABLE 2: Comparison of different types of T cell counts before versus after treatment.

\begin{tabular}{|c|c|c|c|c|c|c|}
\hline & \multicolumn{3}{|c|}{ AD remission after treatment $(N=40)$} & \multicolumn{3}{|c|}{ AD still active after treatment $(N=39)$} \\
\hline & Before & After & $P$ & Before & After & $P$ \\
\hline \multicolumn{7}{|l|}{ CD4+CD25+FoxP3+Tregs } \\
\hline Absolute count $\left(\times 10^{7} / \mathrm{L}\right)$ & $2.06 \pm 1.00$ & $1.03 \pm 0.45$ & $<0.001$ & $2.04 \pm 0.90$ & $2.05 \pm 0.96$ & 0.965 \\
\hline$\%$ in CD4+ cells (\%) & $16.97 \pm 5.01$ & $10.72 \pm 4.87$ & $<0.001$ & $16.76 \pm 4.55$ & $16.77 \pm 4.07$ & 0.994 \\
\hline \multicolumn{7}{|l|}{ CD4+CD25+FoxP3-Teffs } \\
\hline Absolute count $\left(\times 10^{7} / \mathrm{L}\right)$ & $9.78 \pm 3.35$ & $8.10 \pm 3.80$ & 0.034 & $9.98 \pm 3.92$ & $9.97 \pm 4.72$ & 0.993 \\
\hline$\%$ in CD4+ cells (\%) & $82.65 \pm 11.55$ & $76.58 \pm 12.06$ & 0.02 & $82.03 \pm 17.79$ & $81.32 \pm 19.11$ & 0.813 \\
\hline \multicolumn{7}{|l|}{ CD4+IL-10+Tregs } \\
\hline Absolute count $\left(\times 10^{9} / \mathrm{L}\right)$ & $0.26 \pm 0.20$ & $0.22 \pm 0.29$ & 0.501 & $0.26 \pm 0.34$ & $0.26 \pm 0.30$ & 0.941 \\
\hline$\%$ in CD $4+$ cells $(\%)$ & $12.44 \pm 0.42$ & $12.36 \pm 0.38$ & 0.336 & $12.45 \pm 0.54$ & $12.42 \pm 0.38$ & 0.804 \\
\hline
\end{tabular}

The proportion of peripheral CD4+CD25+Tregs in total $\mathrm{CD} 4+\mathrm{T}$ cells in the blood of a healthy person is $5 \%-10 \%$ [28]. Approximately $97 \%$ of Foxp3 is expressed in CD4+CD25+ Tregs. Our current study showed that in patients with AD remission, the proportion of CD4+CD25+FoxP3+Tregs before and after treatment was $16.97 \%$ and $10.72 \%$, respec- tively, indicating the abnormal immune function in patients with $\mathrm{AD}$. In addition, both the pretreatment and posttreatment CD4+CD25+FoxP3+Treg percentages of this current study were higher than the previously reported normal range [28]. These findings appear to be opposite to some previous reports showing that $\mathrm{CD} 4+\mathrm{CD} 25+\mathrm{FoxP} 3+$ Treg proportions 


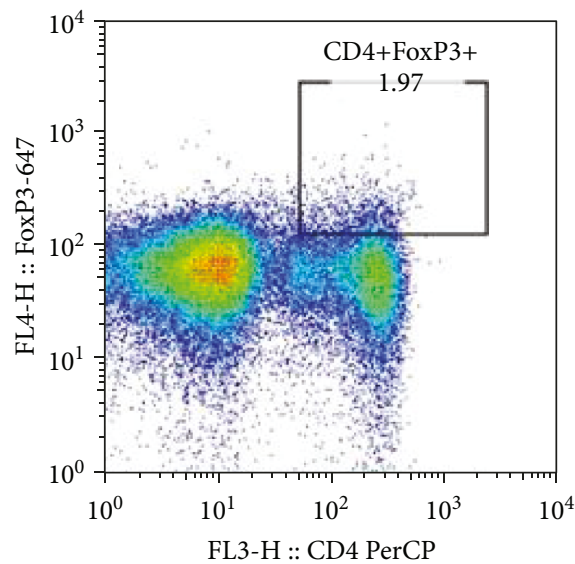

(a)

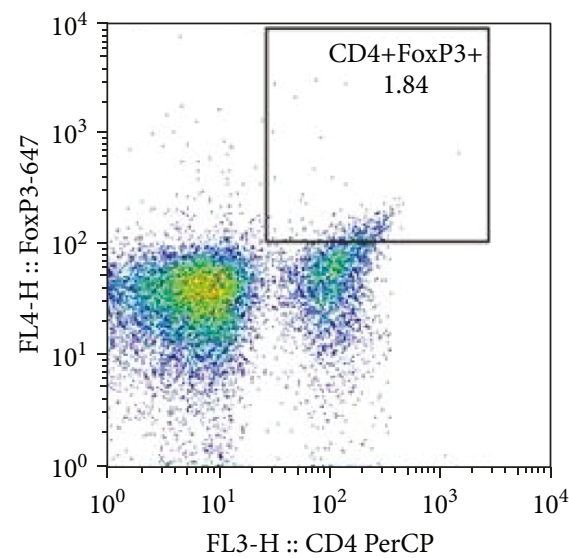

(c)

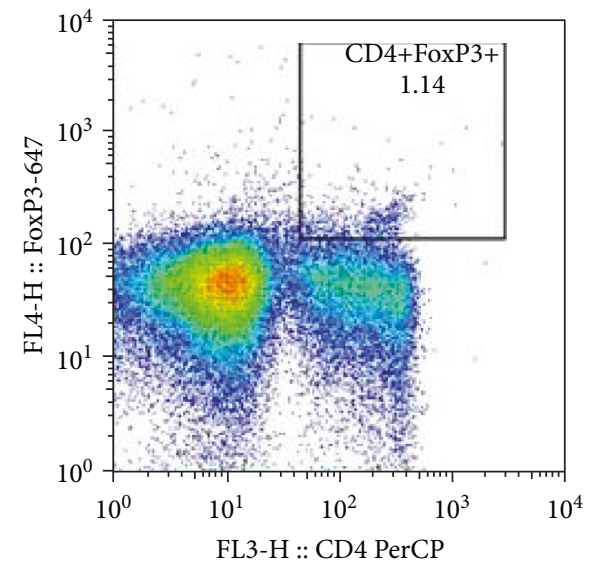

(b)

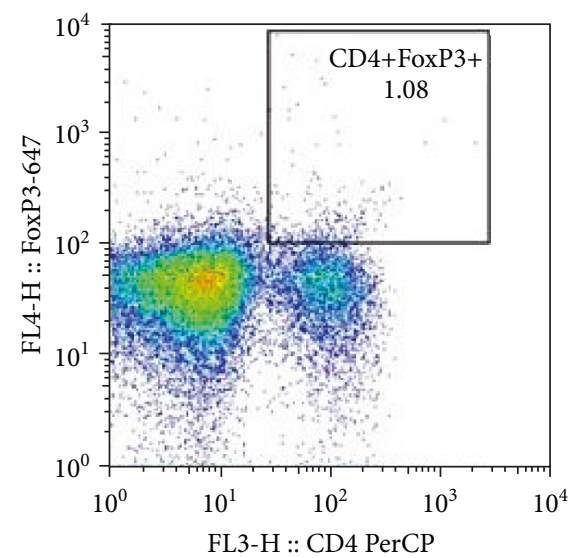

(d)

FIgURE 2: Representative scatter plot of CD4+CD25+FoxP3+Treg analysis. The pretreatment (a) and posttreatment (b) scatter plot of a representative patient from the AD remission group. The pretreatment (c) and posttreatment (d) scatter plot of a representative patient from the $\mathrm{AD}$ active group.

were lower in patients with $\mathrm{AD}$ than in healthy persons $[29,30]$. During the development of AD, elevation of circulating $\mathrm{CD} 4+\mathrm{CD} 25+\mathrm{FoxP} 3+$ Tregs could possibly inhibit severe inflammation in patients with $\mathrm{AD}$. Tregs can inhibit Teff activation via a direct cell-cell contact and cytokines and can also downregulate immune response via the antiinflammatory cytokines IL-10 and TGF- $\beta$. The current study showed that in patients with posttreatment remission, their cell counts of both CD4+CD25+FoxP3+Tregs and CD4+ CD25+FoxP3-Teffs were higher at disease active stage than at remission stage. These results indicate that immune reactions may reduce Treg-mediated inhibition on Teffs as AD progresses, resulting in Teff elevation [28]. Both pretreatment and posttreatment CD4+IL-10+Treg proportions of this current study were higher than the normal range $\left(0.01 \times 10^{9}-0.03 \times 10^{9} / \mathrm{L}\right)$, suggesting that CD4+IL-10+Tregs could be involved in the development of AD. Notably, we found that treatment did not affect CD4+IL-10+Treg cell counts in both remission and active groups, indicating that CD4+IL-10+Treg levels may not correlate with AD status.

The current study showed that in patients with remission, CD4+CD25+FoxP3+Tregs levels reduced after treat- ment but serum levels of IL-10 and TGF- $\beta$, which were mainly secreted by CD4+CD25+FoxP3+Tregs, were not changed after treatment. These results may indicate that the function of $\mathrm{CD} 4+\mathrm{CD} 25+\mathrm{FoxP} 3+$ Tregs could be changed during the development of $\mathrm{AD}$. At $\mathrm{AD}$ onsets, $\mathrm{CD} 4+$ CD25+FoxP3+Tregs may be activated and transported to the target tissue and differentiated. However, their function could be inhibited as $\mathrm{AD}$ exacerbates [5]. In patients with active $\mathrm{AD}$ after treatment, all the three types of Tregs were not affected by treatment, further implying the heterogeneity of $\mathrm{AD}$.

The pretreatment cell count of $\mathrm{CD} 4+\mathrm{CD} 25+\mathrm{FoxP} 3+$ Tregs positively correlated with their pretreatment EASI in all AD patients $(r=-0.482, P \leq 0.001)$. Similarly, previous studies have also found a positive linear correlation between the cell count of CD4+CD25+FoxP $3+$ Tregs and disease severity $[10,11,18,19]$. Subsequently, these AD patients were divided into remission group and active group according to the treatment effect. In the current study, we found that in patients with posttreatment remission, pretreatment cell count of CD4+CD25+FoxP3+Tregs and EASI had a positive linear correlation, indicating that 

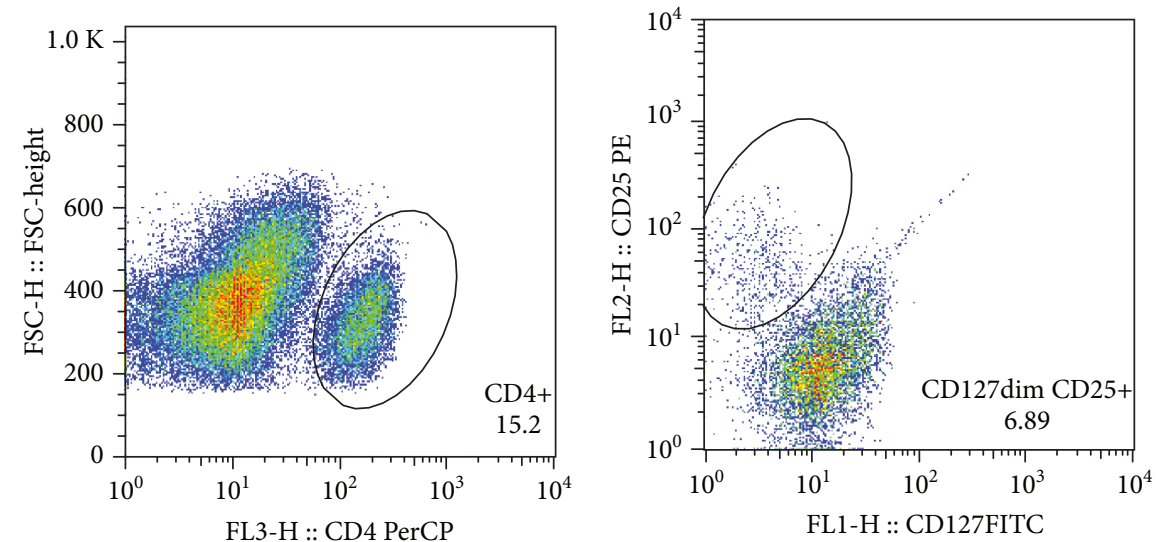

(a)
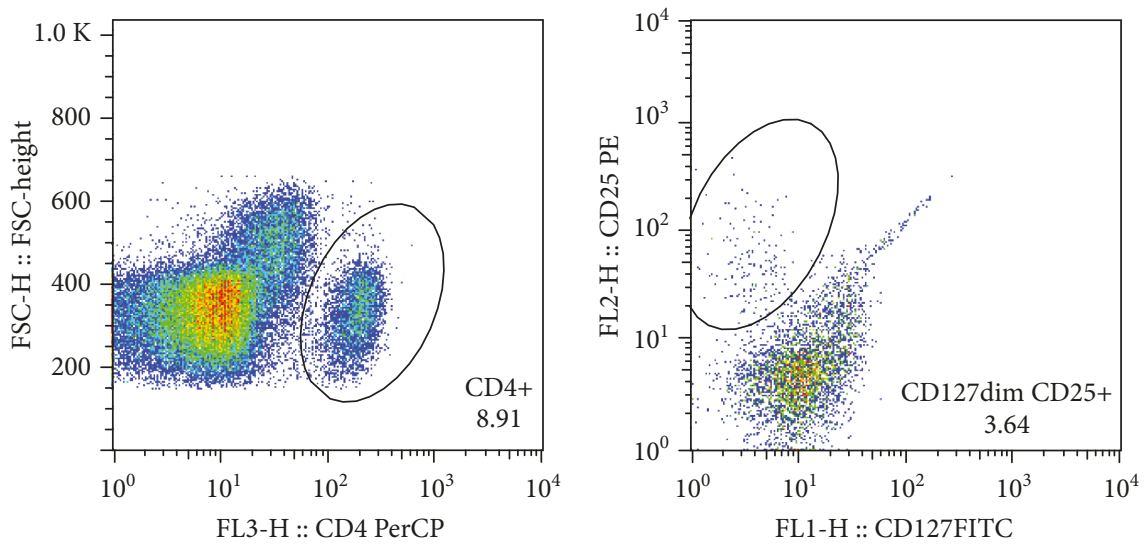

(b)
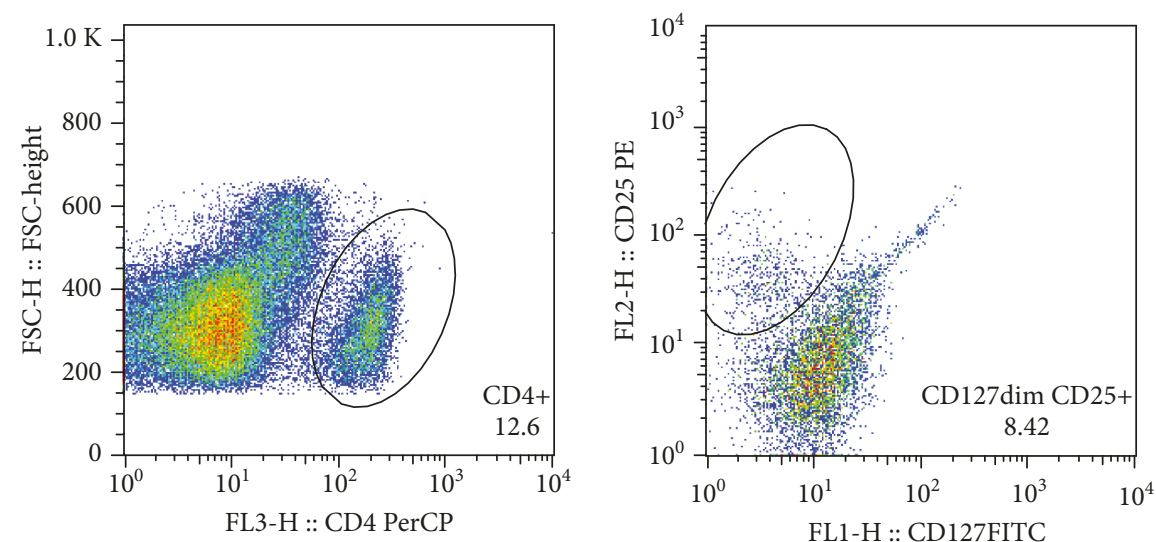

(c)

Figure 3: Continued. 

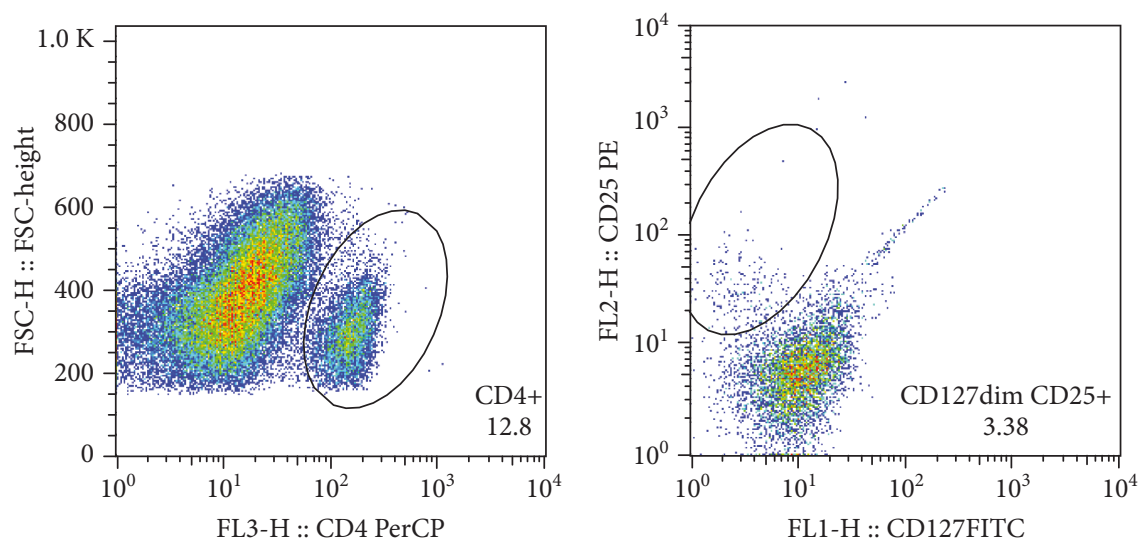

(d)

Figure 3: Representative scatter plot of CD4+CD25+FoxP3-Teff analysis. The pretreatment (a) and posttreatment (b) scatter plot of a representative patient from the $\mathrm{AD}$ remission group. The pretreatment (c) and posttreatment (d) scatter plot of a representative patient from the $\mathrm{AD}$ active group.

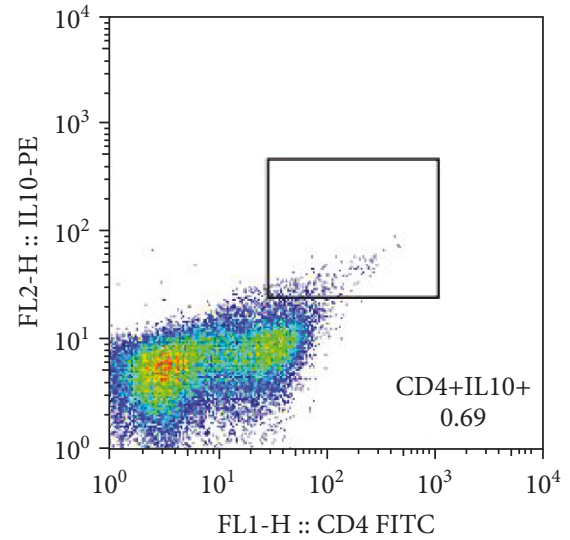

(a)

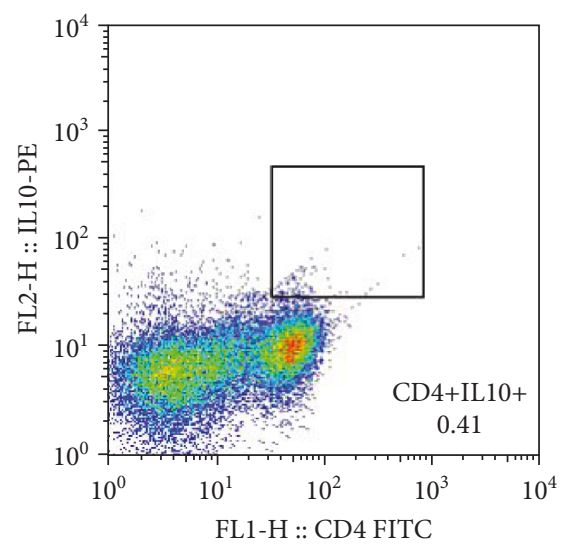

(c)

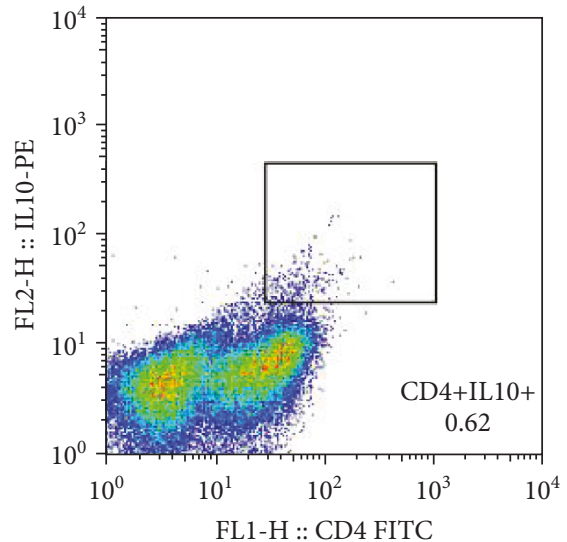

(b)

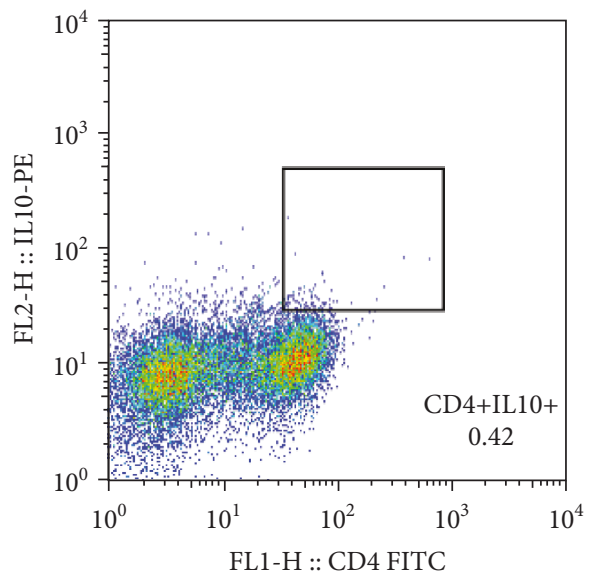

(d)

FIGURE 4: Representative scatter plot of CD4+IL-10+Treg analysis. The pretreatment (a) and posttreatment (b) scatter plot of a representative patient from the $\mathrm{AD}$ remission group. The pretreatment (c) and posttreatment (d) scatter plot of a representative patient from the $\mathrm{AD}$ active group. 

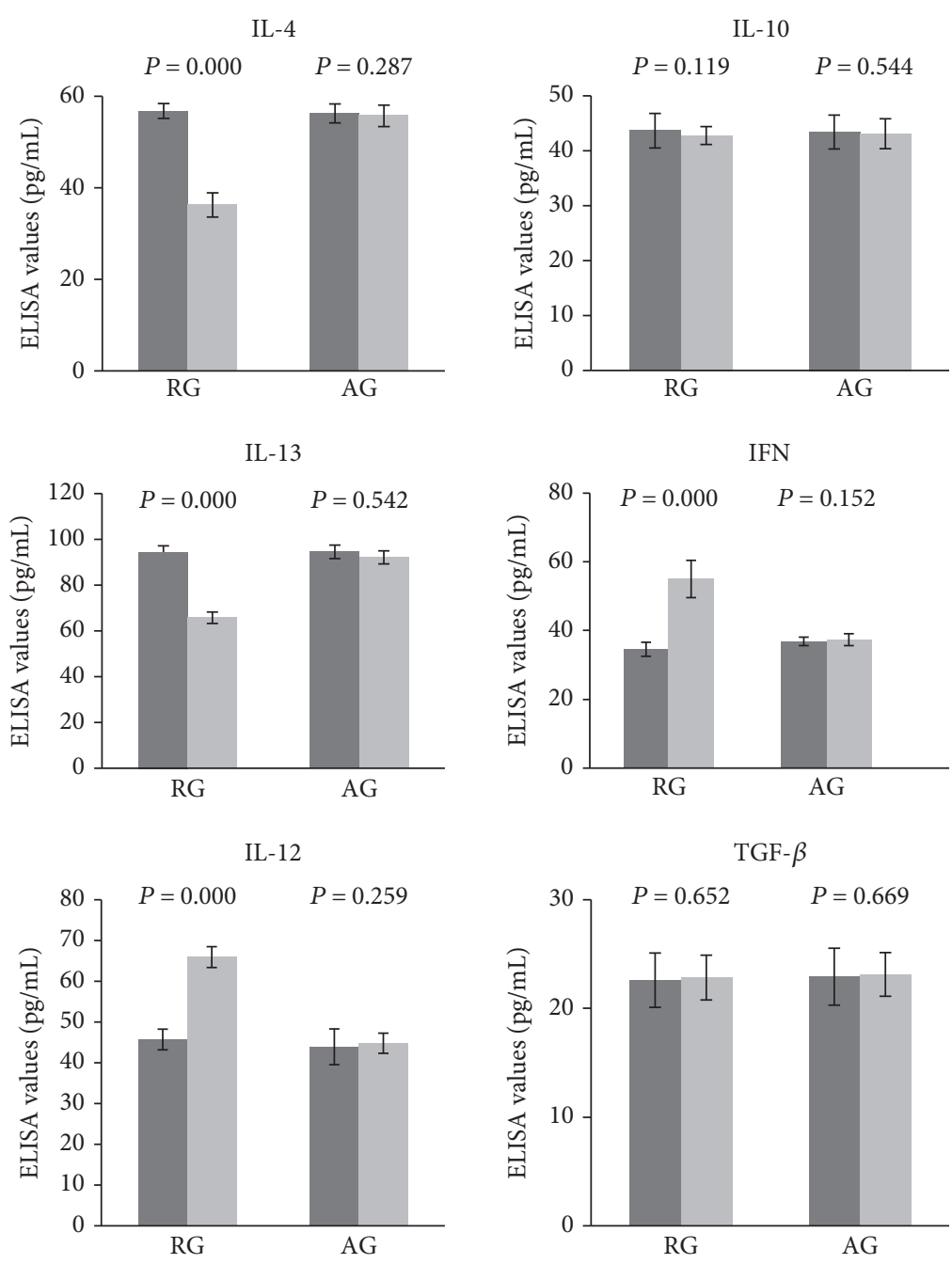

BT: before treatment

AT: after treatment

RG: remission group

AG: active group

Figure 5: Comparison of cytokine levels in serum before versus after treatment. Cytokines in serum samples were analyzed by ELISA. A paired $t$-test was used to compare cytokine levels before versus after treatment.

severer skin lesions appear to be associated with more CD4+CD25+FoxP3+Tregs. However, we found no such correlation in patients with active $\mathrm{AD}$ after treatment. These findings further reflect the complexity of the etiology of $\mathrm{AD}$. CD4+CD25+FoxP3+Tregs may be involved in $\mathrm{AD}$ development in some patients but not in others. We take the view this explains why the results of quite a number of articles appear controversial, or even completely opposite. The reason may very likely be that the target patients have different pathogenesis. AD may be classified into multiple subtypes when diagnosis is based on clinical symptoms. No common biomarkers of $\mathrm{AD}$ have been found yet. Patients diagnosed with $\mathrm{AD}$ can have very different pathogenic mechanisms. Currently, $\mathrm{AD}$ is classified into exogenous $\mathrm{AD}$ and endogenous $\mathrm{AD}$. Our current study also supports the diversity of AD. After all, AD with different pathogenesis could be different diseases and should be treated differently.

In summary, our current study demonstrated that the pretreatment number of $\mathrm{CD} 4+\mathrm{CD} 25+\mathrm{FoxP} 3+$ Tregs was positively associated with $\mathrm{AD}$ severity and reduced significantly at $\mathrm{AD}$ remission in patients with posttreatment remission but not in patients with posttreatment active $\mathrm{AD}$. These data suggest that $\mathrm{AD}$ may be classified into a CD4+CD25+FoxP3+Treg-associated subtype and a nonassociated subtype.

Patients with CD4+CD25+FoxP3+Treg-associated subtype may benefit from conventional treatments better than those with nonassociated subtype.

If this inference could be set up, it will be possible to estimate the curative effect of the $\mathrm{AD}$ patients who have been treated with traditional therapy for a period of time 
by calculating the correlation EASI scores and the number of $\mathrm{CD} 4+\mathrm{CD} 25+\mathrm{FoxP} 3+$ Tregs before and after treatment for these patients. It will be possible to monitor the conditions of CD4+CD25+FoxP3+Treg-associated subtype patients by regularly testing the number of $\mathrm{CD} 4+\mathrm{CD} 25$ + FoxP3+Tregs. The main limitation of this study is the relatively small sample size. Large-scale studies are required to further verify the conclusions.

\section{Data Availability}

The data used to support the findings of this study are included within the article.

\section{Ethical Approval}

The study is approved by the Institutional Review Board of Beijing Friendship Hospital (Approval No. 2015-P2-066-01).

\section{Conflicts of Interest}

All authors declare that there is no conflict of interest.

\section{Authors' Contributions}

Yan Li and Wei Xu contributed equally to this work.

\section{Acknowledgments}

This work was supported by the National Natural Science Foundation of China (No. 81541162). Thanks are due to Dong Zhang for the assistance with experiments and valuable discussion.

\section{References}

[1] Y. Renert-Yuval and E. Guttman-Yassky, "Systemic therapies in atopic dermatitis: the pipeline," Clinics in Dermatology, vol. 35, no. 4, pp. 387-397, 2017.

[2] D. Weiss, M. Schaschinger, R. Ristl et al., "Ustekinumab treatment in severe atopic dermatitis: down-regulation of T-helper 2/22 expression," Journal of the American Academy of Dermatology, vol. 76, no. 1, pp. 91-97.e3, 2017.

[3] M. Sullivan and N. B. Silverberg, "Current and emerging concepts in atopic dermatitis pathogenesis," Clinics in Dermatology, vol. 35, no. 4, pp. 349-353, 2017.

[4] L. F. Eichenfield, W. L. Tom, S. L. Chamlin et al., "Guidelines of care for the management of atopic dermatitis," Journal of the American Academy of Dermatology, vol. 70, no. 2, pp. 338-351, 2014.

[5] L. M. Roesner, S. Floess, T. Witte, S. Olek, J. Huehn, and T. Werfel, "Foxp3(+) regulatory T cells are expanded in severe atopic dermatitis patients," Allergy, vol. 70, no. 12, pp. 16561660, 2015.

[6] V. Moosbrugger-Martinz, C. H. Tripp, B. E. Clausen, M. Schmuth, and S. Dubrac, "Atopic dermatitis induces the expansion of thymus-derived regulatory $\mathrm{T}$ cells exhibiting a Th2-like phenotype in mice," Journal of Cellular and Molecular Medicine, vol. 20, no. 5, pp. 930-938, 2016.

[7] V. Hauck, P. Hügli, M. L. Meli et al., "Increased numbers of FoxP3-expressing CD4+ CD25+ regulatory T cells in periph- eral blood from dogs with atopic dermatitis and its correlation with disease severity," Veterinary Dermatology, vol. 27, no. 1, 2016.

[8] S. Barath, M. Aleksza, T. Tarr, S. Sipka, G. Szegedi, and E. Kiss, "Measurement of natural (CD4+CD25high) and inducible (CD4+IL-10+) regulatory $\mathrm{T}$ cells in patients with systemic lupus erythematosus," Lupus, vol. 16, no. 7, pp. 489-496, 2007.

[9] B. Nedoszytko, M. Lange, M. Sokołowska-Wojdyło et al., "The role of regulatory $\mathrm{T}$ cells and genes involved in their differentiation in pathogenesis of selected inflammatory and neoplastic skin diseases. Part I: Treg properties and functions," Advances in Dermatology and Allergology, vol. 4, no. 4, pp. 285-294, 2017.

[10] K. Gáspár, S. Baráth, G. Nagy et al., "Regulatory T-cell subsets with acquired functional impairment: important indicators of disease severity in atopic dermatitis," Acta Dermato-Venereologica, vol. 95, no. 2, pp. 151-155, 2015.

[11] L. S. Ou, E. Goleva, C. Hall, and D. Y. M. Leung, "T regulatory cells in atopic dermatitis and subversion of their activity by superantigens," The Journal of Allergy and Clinical Immunology, vol. 113, no. 4, pp. 756-763, 2004.

[12] A. J. Reefer, S. M. Satinover, M. D. Solga et al., "Analysis of CD25hiCD4+ "regulatory" T-cell subtypes in atopic dermatitis reveals a novel $\mathrm{T}(\mathrm{H}) 2$-like population," The Journal of Allergy and Clinical Immunology, vol. 121, no. 2, pp. 415-422.e3, 2008.

[13] Y. T. Lin, C. T. Wang, P. S. Chao et al., "Skin-homing CD4+ Foxp3+ T cells exert Th2-like function after staphylococcal superantigen stimulation in atopic dermatitis patients," Clinical and Experimental Allergy, vol. 41, no. 4, pp. 516-525, 2011.

[14] J. M. Hanifin, M. Thurston, M. Omoto et al., "The eczema area and severity index (EASI): assessment of reliability in atopic dermatitis," Experimental Dermatology, vol. 10, no. 1, pp. 11-18, 2001.

[15] Y. Li, W. Xu, and L. Li, "Efficacy and safety of halometasone cream to treat chronic generalized eczema and the effects of halometasone cream on serum cortisol levels," BioMed Research International, vol. 2017, Article ID 3265024, 7 pages, 2017.

[16] A. Szegedi, S. Baráth, G. Nagy et al., "Regulatory T cells in atopic dermatitis: epidermal dendritic cell clusters may contribute to their local expansion," The British Journal of Dermatology, vol. 160, no. 5, pp. 984-993, 2009.

[17] C. Brandt, V. Pavlovic, A. Radbruch, M. Worm, and R. Baumgrass, "Low-dose cyclosporine A therapy increases the regulatory $\mathrm{T}$ cell population in patients with atopic dermatitis," Allergy, vol. 64, no. 11, pp. 1588-1596, 2009.

[18] A. Lesiak, P. Smolewski, D. Sobolewska-Sztychny, A. SysaJedrzejowska, and J. Narbutt, "The role of T-regulatory cells and Toll-like receptors 2 and 4 in atopic dermatitis," Scandinavian Journal of Immunology, vol. 76, no. 4, pp. 405410, 2012.

[19] Z. Samochocki, M. Alifier, P. Bodera et al., "T-regulatory cells in severe atopic dermatitis: alterations related to cytokines and other lymphocyte subpopulations," Archives of Dermatological Research, vol. 304, no. 10, pp. 795-801, 2012.

[20] M. H. El Samahy, E. A. S. Attia, A. A. Saad, and E. Y. Mahmoud, "Circulating CD4(+) CD25(high) FoxP3(+) Tregulatory cells in patients with atopic dermatitis after narrowband-ultraviolet B phototherapy," International Journal of Dermatology, vol. 54, no. 10, pp. e424-e429, 2015. 
[21] Y. J. Choi, H. J. Lee, D. H. Lee et al., “Therapeutic effects and immunomodulation of Suanbo mineral water therapy in a murine model of atopic dermatitis," Annals of Dermatology, vol. 25, no. 4, pp. 462-470, 2013.

[22] J. Udkoff, A. Waldman, J. Ahluwalia, J. Borok, and L. F. Eichenfield, "Current and emerging topical therapies for atopic dermatitis," Clinics in Dermatology, vol. 35, no. 4, pp. 375-382, 2017.

[23] W. E. Paul, "History of interleukin-4," Cytokine, vol. 75, no. 1, pp. 3-7, 2015.

[24] E. Vakirlis, E. Lazaridou, T. G. Tzellos, S. Gerou, D. Chatzidimitriou, and D. Ioannides, "Investigation of cytokine levels and their association with SCORAD index in adults with acute atopic dermatitis," Journal of the European Academy of Dermatology and Venereology, vol. 25, no. 4, pp. 409416, 2011.

[25] A. Lesiak, M. Zakrzewski, K. Przybyłowska, M. RogowskiTylman, A. Wozniacka, and J. Narbutt, "Atopic dermatitis patients carrying $\mathrm{G}$ allele in -1082 G/A IL-10 polymorphism are predisposed to higher serum concentration of IL-10," Archives of Medical Science, vol. 10, no. 6, pp. 1239-1243, 2014.

[26] P. Y. Hussein, F. Zahran, A. Ashour Wahba et al., "Interleukin 10 receptor alpha subunit (IL-10RA) gene polymorphism and IL-10 serum levels in Egyptian atopic patients," Journal of Investigational Allergology \& Clinical Immunology, vol. 20, no. 1, pp. 20-26, 2010.

[27] W. Jin, H. U. Boying, M. A. Xinhua, S. Chen, and Y. Cao, "Detection of interleukin-10 in sera and culture supernatants of CD4+CD25+T cells from children with atopic dermatitis," Chinese Journal of Dermatology, vol. 46, no. 1, pp. 13-15, 2013.

[28] Y. Y. Zhang, A. X. Wang, L. Xu, N. Shen, J. Zhu, and C. X. Tu, "Characteristics of peripheral blood CD4+CD25+ regulatory $\mathrm{T}$ cells and related cytokines in severe atopic dermatitis," European Journal of Dermatology, vol. 26, no. 3, pp. 240-246, 2016.

[29] L. Ma, H. B. Xue, X. H. Guan et al., "The imbalance of Th17 cells and CD4(+) CD25(high) Foxp3(+) Treg cells in patients with atopic dermatitis," Journal of the European Academy of Dermatology and Venereology, vol. 28, no. 8, pp. 1079-1086, 2014.

[30] A. Stelmaszczyk-Emmel, A. Zawadzka-Krajewska, A. Szypowska, M. Kulus, and U. Demkow, "Frequency and activation of CD4+CD25 FoxP3+ regulatory T cells in peripheral blood from children with atopic allergy," International Archives of Allergy and Immunology, vol. 162, no. 1, pp. 16$24,2013$. 


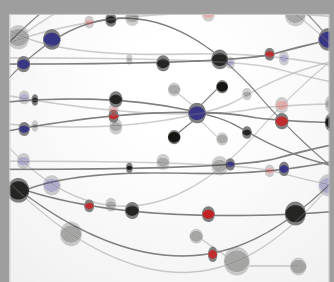

The Scientific World Journal
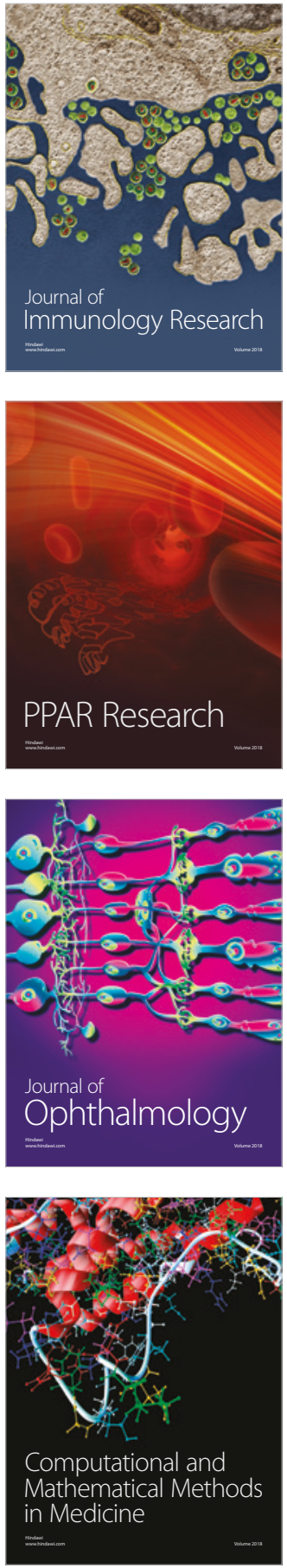

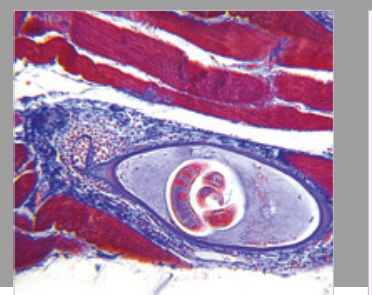

Gastroenterology Research and Practice

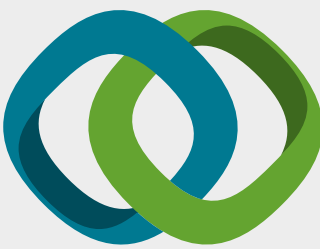

\section{Hindawi}

Submit your manuscripts at

www.hindawi.com
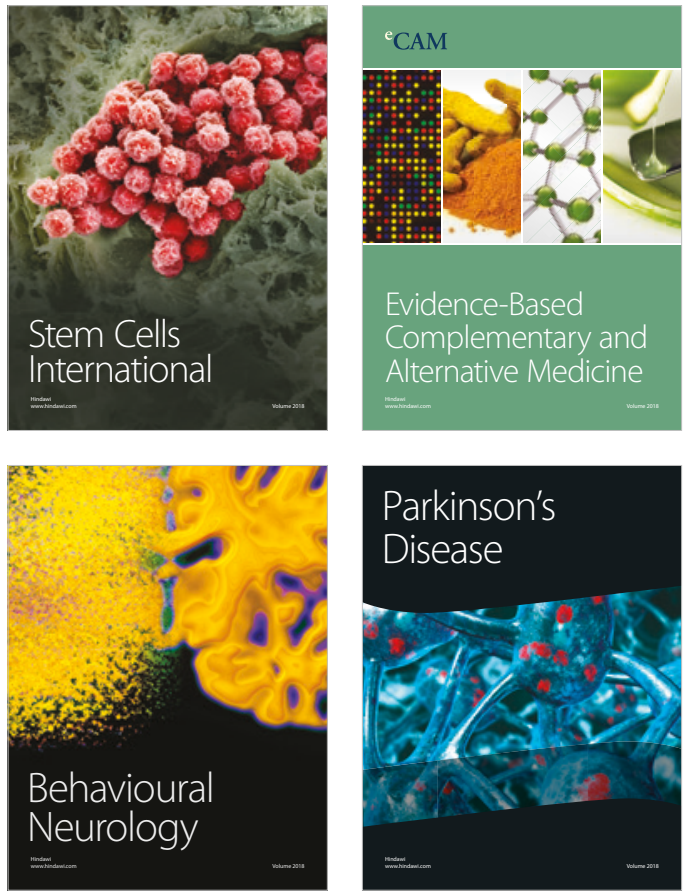

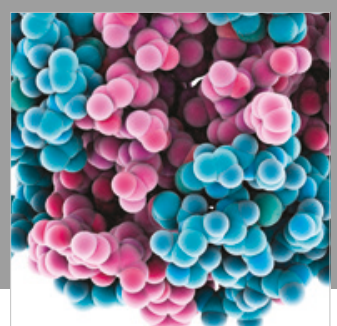

ournal of

Diabetes Research

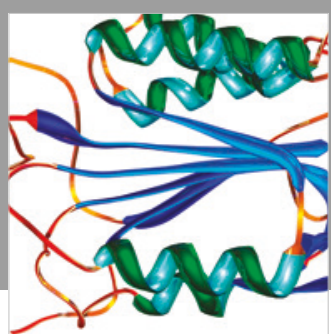

Disease Markers
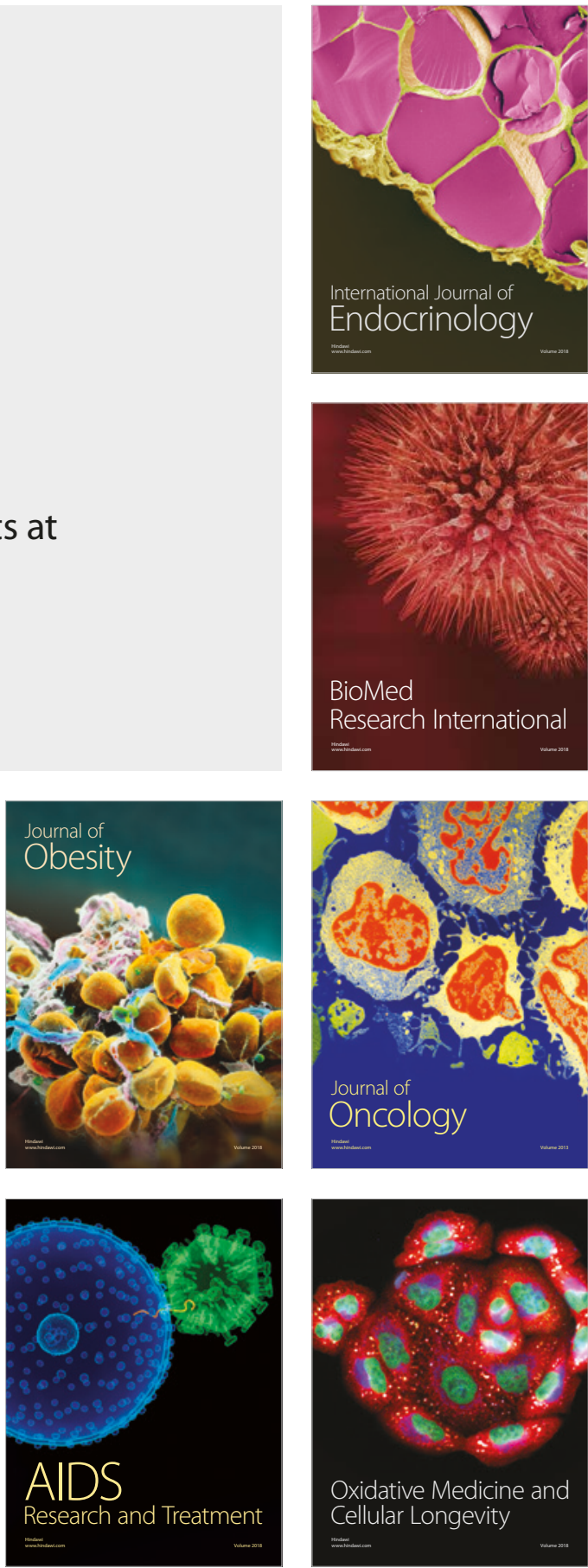